\title{
LA CONSTANTE LUCHA DE LA CONSTITUCIÓN POLÍTICA: CONTROLAR EL PODER DEL SOBERANO
}

THE POLITICAL CONSTITUTION'S CONSTANT STRUGGLE: TO CONTROL THE POWER OF THE SOVEREIGN

Alejandro Guevara Arroyo ${ }^{1}$

\section{RESUMEN:}

Este breve texto es una introducción filosófica a una de las funciones principales de la Constitución Política (en acuerdo con el modelo liberal). Se trata de la función de control del poder político. Adicionalmente, se presentan varios mecanismos que se han desarrollado para darle efectividad a la Constitución, así como las variopintas dificultades con las que se enfrenta.

PALABRAS CLAVE: Filosofía del derecho, derecho constitucional, constitución política, poder político, control del poder, división de poderes, balanceo de pesos y contrapesos, y tribunal constitucional.

\section{ABSTRACT:}

This brief paper is a philosophical introduction to one of the Constitution's main functions (in accordance with the liberal model). That is the function of control of the political power. Moreover, it presents several mechanisms that have been developed to give effectivity to the Constitution, as well as the various difficulties it faces.

KEYWORDS: philosophy of law, constitutional law, Political Constitution, political power, power control, power division, check and balances, constitutional court.

\section{Recibido: 16 de julio 2018}

Aceptado: 07 de agosto 2018

1 Profesor de la Universidad de Costa Rica; maestrando en Filosofía del Derecho por la Universidad de Buenos Aires, Argentina; doctorando en Filosofía del Derecho por la Universidad de Buenos Aires, Argentina; integrante de la Asociación Costarricense de Filosofía del Derecho y Filosofía Práctica; estudiante avanzado de Filosofía por la Universidad de Costa Rica; alguear7@gmail.com 


\section{INTRODUCCIÓN}

Hace más de una década los filósofos latinoamericanos Ricardo Guibourg y Daniel Mendonca publicaron un libro dedicado a la filosofía del derecho constitucional. La obra lleva un título espléndido: La odisea constitucional. Como Ulises en su navío, las sociedades contemporáneas confían en que cierta creación jurídica les brindará protección en su travesía histórica. Se trata de un texto -ni más ni menos- con un especial lugar (i.e. una especial jerarquía) en los ordenamientos jurídicos: la Constitución Política.

A continuación, se analizará una de las más valiosas funciones reales de dicho navío: la de controlar el poder del soberano. También señalaré someramente los medios con que las sociedades occidentales disponen para cumplir dicha función. Finalmente, mencionaré algunos de sus problemas y limitantes.

\section{METODOLOGÍA}

Este documento presenta un análisis teórico tanto de una institución jurídico-política, como de la concepción teórica que la acompaña. En otras palabras, este texto se dedica a la reconstrucción y análisis de las relaciones inferenciales que existen entre distintas tesis y argumentos. Los temas y problemas que se abordarán son -casi sobra decirlo- cuestiones que lindan entre la ius-filosofía y la filosofía política y del Estado.

Se presupone una cierta forma de hacer filosofía: la analítica. En otras palabras, una clase de filosofar dedicada, en primer lugar, a precisar y aclarar los significados de términos relevantes dentro de cierto entramado teórico y a reconstruir las redes inferenciales existentes en las retículas de enunciados que conforman teorías y concepciones. En segundo lugar, esta clase de filosofar también permite la crítica racional de la verdad o idoneidad de las tesis analizadas y de la validez inferencial de los argumentos reconstruidos.

\section{La Constitución, un navío inestable}

Podemos identificar muchos cometidos para las Constituciones Políticas. Acá se pondrá énfasis en uno solo de ellos: el de limitar el poder ${ }^{1}$ político del soberano (o de quien se considere su representante) ${ }^{2}$. Este es, de hecho, uno de los empleos primigenios de las Constituciones (por ejemplo, en la Constitución de los Estados Unidos de América de 1787 o -aun antes- en la Carta de Juan I de Inglaterra de 1215). En otras palabras: mediante las Constituciones Políticas se intenta limitar lo que las personas con el poder político soberano pueden hacer y mandar a hacer. Ahora, esta función es valiosa para quienes les preocupa el rebalse del poder político del soberano sobre su población, las minorías y los individuos (la minoría absoluta). En la historia del pensamiento, esta preocupación es una de las características típicas de las concepciones del liberalismo político.

Incluso, el sentido del término Constitución en una parte de la filosofía política es justamente ese: un ordenamiento jurídico (sea un texto o no) de especial jerarquía en que se encuentran limitaciones del poder del soberano sobre los ciudadanos. Es sintomático lo enunciado en el artículo 16 de la Declaración de los derechos del hombre y del ciudadano (1789): "Una sociedad en la que no esté asegurada la garantía de los derechos ni reconocida

1 Aquí se utilizará el término poder en un sentido semejante al del agudo filósofo inglés B. A. W. Russell (en su 2017). Por la palabra poder se puede entender (a) la producción o alcance de los efectos deseados o buscados o (b) los medios efectivos para lograr dicha producción.

2 En la erudita reconstrucción histórica de Fioravanti, esta es una de las dos concepciones fundamentales sobre las funciones de las constituciones políticas a través de la modernidad y la contemporaneidad. La segunda concepción afirma que la Constitución Política es la instancia donde se manifiesta la voluntad del soberano. Fioravanti le llama a la primera modelo liberal (o republicano). La segunda es el modelo radical. Ambos modelos tienen distintas justificaciones políticas. Además, en distintos respectos, las normas que siguen estos diversos modelos pueden tener incompatibilidades prácticas entre sí. Para profundizar las características, transformación, relaciones y manifestaciones históricas de estas dos concepciones, ver, del mencionado autor, Constitucionalismo. Experiencias históricas y tendencias actuales, 59-91. 
la división de poderes, no tiene Constitución" (la cursiva va por mi cuenta) ${ }^{3}$.

Ahora bien, para lograr esto, las Constituciones tienen dos clases de medios o instrumentos: unos internos y otros externos. Sin embargo, también tienen limitantes. Veamos:

(a). Los instrumentos internos consisten en normas jurídicas de varios tipos:

En primer lugar, están aquellas que diluyen los poderes estatales en distintas instituciones y establecen las reglas generales de los procedimientos que estas deben seguir para desarrollar sus actividades. A esto se le llama división de poderes. En segundo lugar, hay normas que disponen en qué respectos y cómo los representantes de distintos poderes del Estado pueden afectarse y estorbarse entre sí: mecanismo de pesos y contrapesos. Finalmente, también se obliga a los poderes políticos a perseguir ciertas finalidades políticas en favor de los ciudadanos. Asimismo, se establecen límites de lo que aquellos pueden hacer y mandar sobre los 'súbditos'. Estas normas son los derechos

3 Existen otros sentidos de la palabra Constitución Política utilizados por juristas, teóricos del derecho, historiadores y teóricos políticos. Así, es habitual entre los juristas e ius-filósofos considerar que Constitución menta un conjunto de normas (o el texto que enuncia un conjunto de normas) de especial jerarquía en el ordenamiento jurídico ( $\mathrm{y}$-en diversos sentidos- fundamental para este). Por otro lado, los doctrinarios del derecho constitucional y los constitucionalistas prácticos le llaman Constitución a cierto texto jurídico en el que se recogería la llamada materia constitucional o el derecho constitucional. Sin embargo, es una construcción doctrinaria aquella que señala, además, que ese texto no necesariamente contiene sólo normas constitucionales, ni colma la totalidad del derecho 'de la Constitución'. Por cierto, esta clasificación de diversos sentidos utilizados habitualmente por distintos usuarios de la palabra Constitución no deben confundirse con varias clases de Constitución, asunto bastante diferente. Por otro lado, cabe tener en cuenta que la clasificación presentada es una simplificación, pues en la vida real distintos hablantes mezclan de distintas formas estos sentidos y otros más. Finalmente, quizás sea de interés rescatar un sentido de la palabra Constitución útil en especial para historiadores: un ordenamiento normativo jurídico-político de las principales relaciones sociales y políticas de una comunidad (o de un conjunto de comunidades). Tal es el sentido que utiliza el historiador M. Fioravantti (en 2011) y le permite hablar de Constituciones incluso en la antigüedad y en el medioevo europeos. fundamentales: personales, políticos y sociales ${ }^{4}$.

En la actualidad comúnmente se denomina parte orgánica a la sección de la Constitución que prescribe la división de poderes y los mecanismos de pesos y contra pesos. Por su parte, se llama parte dogmática a la sección de derechos fundamentales.

Por su puesto, debe evitarse que los que ostentan el poder político cambien fácilmente tales normas. Caso contrario, estas no servirán como medios para controlar al soberano. Debido a ello, es común que las propias Constituciones prohíban que el poder ejecutivo cambie el texto constitucional mediante decretos. Lo mismo es cierto del poder legislativo y sus leyes: está prohibido mediante leyes reformar la Constitución Política. Para cambiar el texto constitucional se ordena un procedimiento agravado y difícil de cumplir. La inexistencia o incumplimiento de estas normas procedimentales es un limitante para a la función de control constitucional del poder político.

(b) Dentro de los mecanismos externos se encuentran las instituciones encargadas de controlar el cumplimiento de lo mandado por la Constitución Política (incluidas las limitaciones del poder del soberano).

Vale la pena recordar que en la historia del pensamiento jurídico existió alguna polémica sobre qué institución estatal debía asumir este papel. Mencionaré -a modo de ejemplificación- dos famosas posiciones que contendieron en el mundo germano a inicios del siglo XX.

En una esquina estaba Carl Schmitt, un famoso jurista y filósofo político que vivió de 1888 a 1985. Schmitt consideró -en su El guardián de la Constitución (Kelsen \& Schmitt, 2015)- que el encargado de controlar el cumplimiento de la Constitución debía ser el propio gobierno (como representante del Pueblo -que considera el soberano real-).

4 Pueden ampliarse las características de estos mecanismos en Guastini, 2016, capítulo XXIX. 
En la otra esquina estaba Hans Kelsen (nacido en Viena 1881), uno de los más importantes filósofos del derecho y de la política del siglo pasado. Frente a Schmitt, Kelsen defendió que un tribunal especializado e independiente de los poderes representativos (i.e. ejecutivo y legislativo) debía encargarse de controlar el cumplimiento de lo mandado por la Constitución. Un tribunal con tal función tendría un papel sin duda político, pero de central importancia para mantener la democracia y la república en los Estados (ver la obra de Kelsen: ¿Quién debe ser el guardián de la Constitución? Kelsen \& Schmitt, 2015- ).

La posición de Schmitt triunfó sobre la de Kelsen en el mundo germano de inicios del siglo XX. Su visión del Estado, de las relaciones políticas y de la sociedad, fue una de las tantas inspiraciones que reclamó para sí el nacional-socialismo. No por nada Schmitt fue considerado el gran jurista del Tercer Reich. Las ideas de Kelsen (en estos y otros asuntos) no solo fueron rechazadas por el régimen, sino que -en conjunto con acusaciones de tener ancestros judíos- obligaron al filósofo vienés a abandonar su país y emigrar a los Estados Unidos de América (en donde falleció, en el año de 1973).

No obstante, pasada la noche del nacionalsocialismo, las democracias occidentales asumieron el control constitucional tal y como lo planteó Kelsen. Así, hoy día en muchos países existen tribunales especializados que se encargan del control constitucional sobre aquellos que ostentan el poder político oficial ${ }^{5}$ (Mendonca \& Guibourg, 2004, cap. VII).

5 Hay indicios de una tendencia real difícil de analizar y valorar: se trata del aumento considerable del poder que sobre el ordenamiento jurídico manejan los propios tribunales constitucionales. Esto puede estar jurídicamente relacionado con la aceptación generalizada de lo que algunos ius-filósofos llaman neo-constitucionalismo (análisis detallado en: Comanducci, 2016). De mi parte, creo que un primer paso para entender esta tendencia es considerar (realistamente) que los tribunales constitucionales poseen dosis de discrecionalidad judicial inusuales comparados con otros jueces y tribunales. $\mathrm{He}$ desarrollado este tema en un artículo titulado Discrecionalidad de los tribunales constitucionales y los principios jurídicos: un análisis realista (pendiente de publicación).

\section{El gran límite del control constitucional}

Ahora bien, dicho todo esto, es obligatorio traer a colación un límite clave para el control constitucional del poder del soberano. Es tan importante que con frecuencia se lo olvida. En palabras de Guibourg y Mendonca: "La fuerza de las normas suele depender, ante todo, de la fuerza con la que los órganos estatales en especial y los ciudadanos en general estén dispuestos a hacerlas cumplir; pero esa es una fuerza ajena a las normas mismas" (2004, pp. 26 -la negrita es mía-).

En otras palabras: si los representantes del poder político, los jueces y los ciudadanos no están más o menos de acuerdo con lo que la Constitución manda -o les importa poco si se cumplen o no sus normasno hay documento (por más bella o sensatamente que esté redactado) que sirva para contener el abuso del poder del soberano ${ }^{6}$. Así, en algún sentido, la potencia con que se limite el poder del soberano mediante la Constitución Política depende en gran medida de la fuerza con que los controlados (soberanos y súbditos) estén dispuestos a aceptar dichas limitaciones ${ }^{7}$.

\section{CONCLUSIONES (Síntesis y excurso conclusivo)}

En las previas líneas se intentó precisar las relaciones teóricas que existen entre la función de controlar el poder del soberano y la Constitución Política. Al tiempo, se precisaron los distintos medios (internos: normas y externos: instituciones)

6 Se trata, al fin y al cabo, de lo que Alf Ross llamó la ideología jurídica: la vivencia de que un régimen efectivo de fuerza es además- normativamente válido u obligatorio. Obviamente, "es imposible [...] distinguir entre un 'orden jurídico' y un 'régimen de violencias', por cuanto la cualidad de la validez [(i.e. su fuerza obligatoria)] que serviría para caracterizar al derecho no es una cualidad objetiva del orden mismo, sino sólo una expresión de la manera en la que éste es vivido (experienced) por los individuos" (Ross, 2011, 83).

7 Un análisis interesante sobre la situación político-constitucional latinoamericana, cómo afecta la función constitucional y sus posibilidades de mejora, en Mendonca \& Guibourg, 2004, cap. IX. 
para lograr este cometido, pero también algunas de sus limitantes y dificultades.

En fin, tal es la perpetua faena del navío/ Constitución: proteger a los ciudadanos del soberano (incluso si este es querido por ellos o se supone que los representa). Empero, un peligro es contante: que los propios marineros y tripulantes agujereen la nave.

\section{BIBLIOGRAFÍA}

- Comanducci, P. (2016). Estudios sobre Constitución y derechos fundamentales. Queretaro: Instituto de Estudios Constitucionales del Estado de Querétaro.

- Fioravanti (2011). Constitución. De la Antigüedad a nuestros días (M. Martinez Neiva). Madrid: Trotta.

- Fioravanti (2014). Constitucionalismo. Experiencias históricas y tendencias actuales. (A. Mora Canada y M. Martinez Neira, trad.). Madrid: Trotta.

- Guastini, R. (2016). La sintaxis del derecho. (A. Núñez Vaquero, trad.) Madrid: Marcial Pons.

- Kelsen \& Schmitt (2015). The Guardian of the Constitution: Hans Kelsen and Carl Schmitt on the Limits of Constitutional Law. (transl. by Lars Vinx with an introd. and notes by Lars Vinx). United Kingdom: Cambridge University.

- Mendonca \& Guibourg (2004). La odisea constitucional. Constitución, teoría y método Madrid: Marcial Pons.

- Ross, A. (2011). Sobre el derecho y la justicia (trad. G. Carrió). Buenos Aires. EUDEBA.
- Russell, B. (2017). El poder: un nuevo análisis social. Barcelona: The Bertrand Russell Peace Foundation. Edition Kindle. 